\title{
A Custom Made Post and Core Technique for Restoring Badly Broken Mandibular Posterior Tooth: A Case Report
}

\author{
Guragain $\mathrm{M}^{1 *}$, Mathema $\mathrm{SRB}^{2}$, Maharjan $\mathrm{SK}^{3}$, Sah $\mathrm{S}^{4}$ \\ ${ }^{1}$ Lecturer, Institute of Medicine, Kathmandu, Nepal \\ ${ }^{2}$ Professor, ${ }^{3}$ Assistant Professor, Department of Prosthodontics and Maxillofacial Prosthetics, \\ People's Dental College and Hospital, Shorakhutte, Kathmandu, Nepal. \\ ${ }^{4}$ Assistant Professor, Dental Department, National Academy of Medical Sciences, Bir Hospital, Kathmandu, Nepal
}

\begin{abstract}
The successful treatment of a badly broken tooth depends both on good endodontic therapy and prosthetic reconstruction of the tooth. Molars with almost total coronal destruction can be salvaged by the use of customized post and core. A number of methods have been suggested for making a cast post and core using two or more of the canals to increase retention of the prosthesis. This case report discusses a method to restore a structurally compromised, endodontically treated mandibular first molar using a customized cast post and core.
\end{abstract}

Key words: Endodontic therapy, Post and core, Molar, Structurally damaged tooth

\section{Introduction}

$\mathrm{E}$ ndodontically treated teeth and its restoration is a topic that has been extensively studied, yet it remains controversial from many perspectives. ${ }^{1}$ The loss of a considerable portion of the clinical crown hinders sufficient retention of restorations from the remaining tooth structure. Thus, in such situations, a root canal-retained restoration is advocated. $^{2}$

The primary function of the post is to provide retention for the core in a tooth with extensive loss of coronal tooth structure. ${ }^{3,4}$ In molars post is placed in the straightest canal. ${ }^{1}$

There are many techniques of restoring a badly broken molar tooth after successful endodontic treatment. Shillingburg, Fisher and Dewhirst

*Corresponding Author

Dr. Manjeev Guragain, Lecturer

Institute of Medicine, Kathmandu, Nepal

E-mail: manjeevguragain@gmail.com presented a technique in which the master post was supplemented with a short key in the second canal. According to the authors, this additional key aids in seating the post and prevents rotation, but it adds little retention. ${ }^{7}$

\section{Case Report}

A forty -year-old male patient reported with complaint of fractured tooth in right lower back teeth region. Clinical and radiographic examination revealed fractured right mandibular first molar with gingival and periodontal status. Occlusion was evaluated and alginate impressions (Zelgan, Dentsply, India) of both upper maxillary and lower mandibular arches were made for making the diagnostic casts.

Complete oral prophylaxis and maintenance protocol, root canal treatment, fabrication of cast post followed by restoration of the tooth with metal crown was planned for the patient. The treatment plan and its advantages and disadvantages were discussed with the patient and an informed consent was obtained before treatment was started. 


\section{Technique}

Endodontic treatment was completed in a conventional way taking into consideration the remaining bone support, root anatomy, root curvature and the apical obturation (Fig 1 and 2). Patient was recalled after three months for evaluation of the endodontic treatment. Being confirmed of the success of the treatment, the procedure for post space preparation was started. A Gates Glidden drill was used to remove guttapercha (GP) from the pulp chamber. GP from the distolingual canal (the most straight canal of the four canals) was removed with peeso reamer (Largo peeso reamers, Dentsply, India) mounted on air motor hand piece to an established length of $10 \mathrm{~mm}$ from the orifice. Similarly, GP from mesiolingual canal was removed to a length of $5 \mathrm{~mm}$ for preparation of the anti-rotational key. Post space was prepared in the distal canal of tooth with Peeso reamer. A Hedstrom-file was used circumferentially to smoothen the preparation of the post spaces (Fig 3 and 4). Radiolucency is present in the bifurcation area. Tooth was roughly prepared for a crown, with equigingival chamfer finish line. The canal was shaped with peeso reamers (Largo peeso reamers, Dentsply, India). All unsupported/ thin enamel and dentine were removed using a horizontally approaching diamond. ${ }^{8}$

Two tapered sticks of approximately the diameter same as those of the canals were made from self-cure resin (RR, Dentsply, India). They were tried in the canals and trimmed until they fit snugly into them. Root canals surface were coated with a suitable lubricant. An autopolymerizing resin (RR, Dentsply, India) were applied on the custom-made acrylic posts using brush bead technique and replaced into the prepared post spaces. Post was slowly withdrawn and then again reseated to prevent binding of acrylic resin into the root canal when the material was in dough stage.

When the acrylic resin achieved an initial set, the pattern was moved in and out of the canal to ensure that it was not locked into undercuts in the canal. As the resin polymerized, the post was removed from the canal, and was confirmed of its extension to the bottom of the preparation (Fig 5).

The post spaces were lubricated and posts were seated in place. Another mix of acrylic resin was mixed and placed on the exposed part of the stick to provide adequate bulk for the preparation for the final restoration. The preparation for the final restoration was completed with the postcore pattern in place. The post-core assembly was then carefully removed from the prepared tooth and stored in distilled water until it was invested and casted in metal alloy.

After getting the cast, fit of the post and core was checked in the tooth by seating it with light pressure. The core portion of the casting was polished and the luting cement (GC, GC Corporation, Tokyo, Japan) was mixed and inserted into the post spaces with a lentulo spiral (Lentulo Spiral filler, Dentsply, India). The post and core was inserted slowly into the canal allowing the excess cement to escape away from the post spaces, allowing the post and core to seat completely (Fig 6). The preparation was finished and impressions were made with light body- putty polyvinyl siloxane impression material (Reprosil, Dentsply, India) for the lower mandibular arch and alginate (Zelgan, Dentsply, India) for the upper maxillary arch. The metal crown was fabricated and was cemented at a subsequent appointments (Fig 7 and 8). 


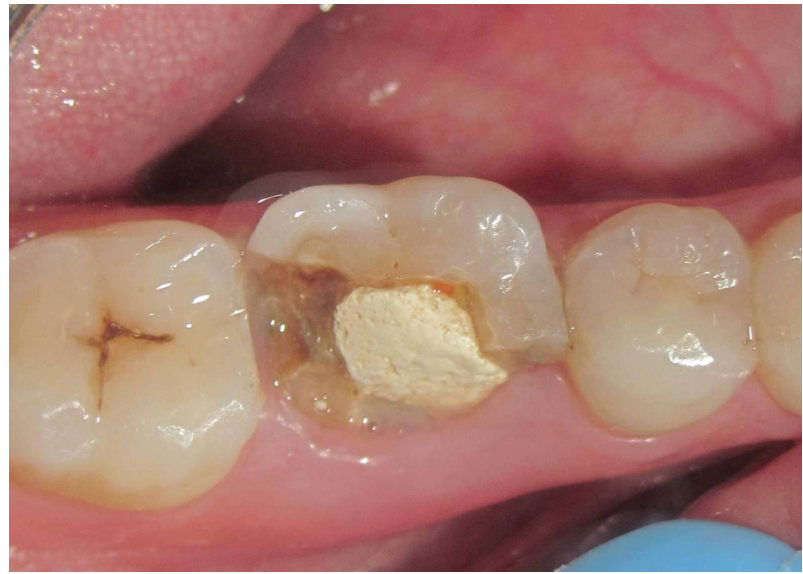

Figure 1: Fractured buccal portion of lower right first molar

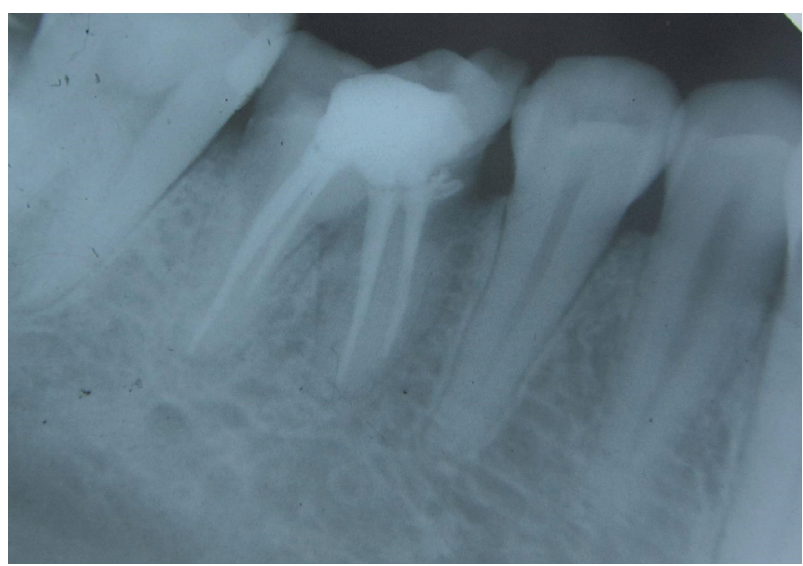

Figure 2: IOPA view of fractured mandibular right first molar

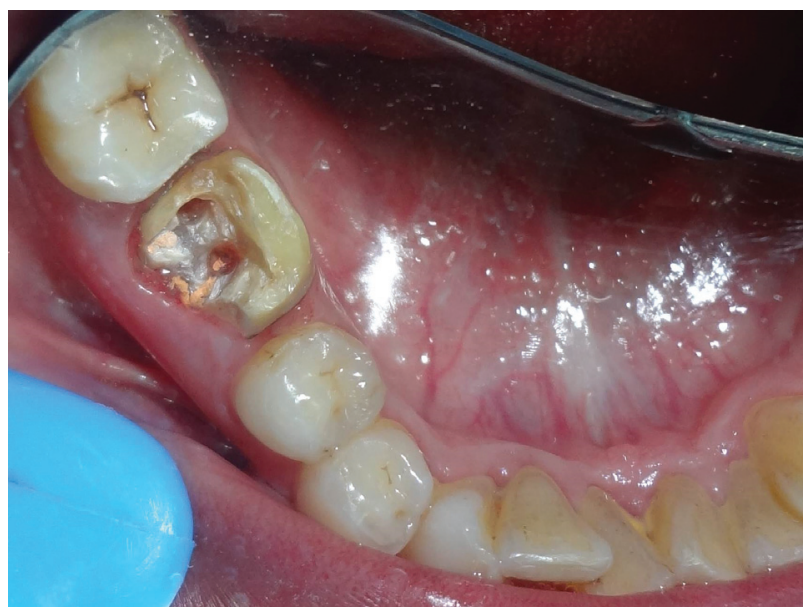

Figure 3: Post space preparation

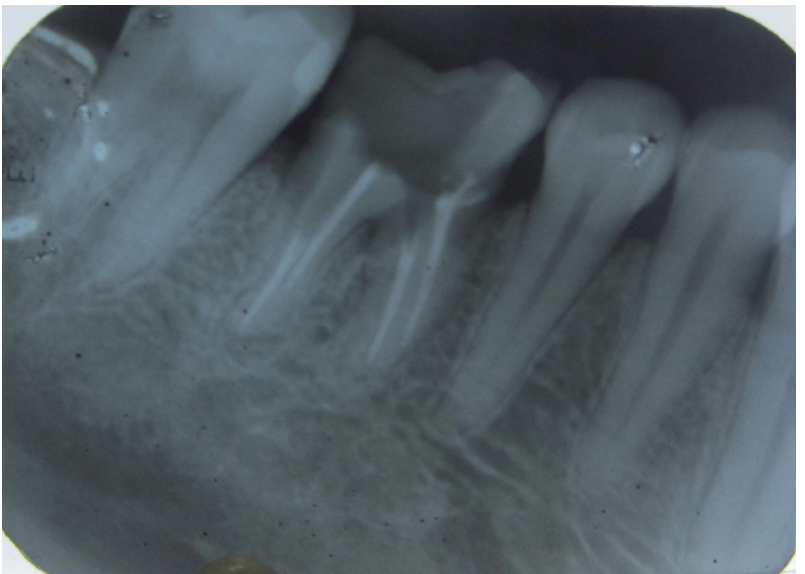

Figure 4: IOPA view after post space preparation

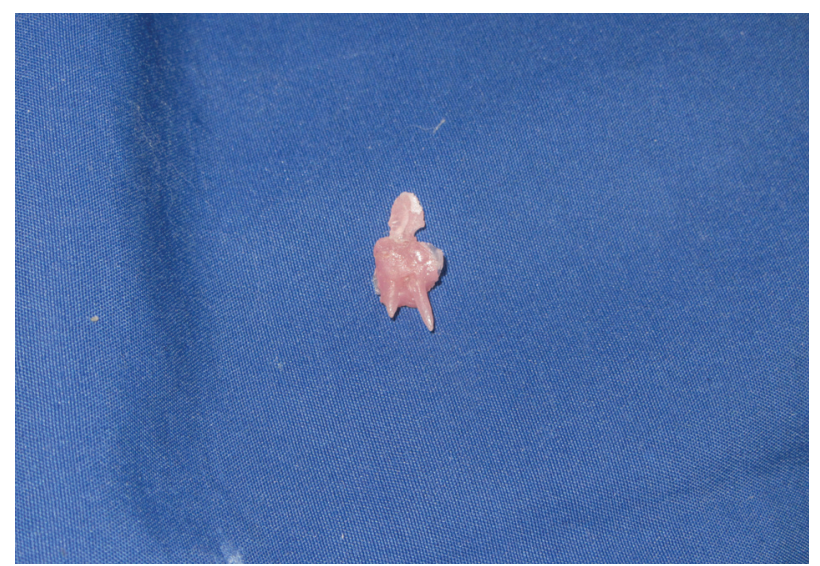

Figure 5: Direct post pattern fabrication

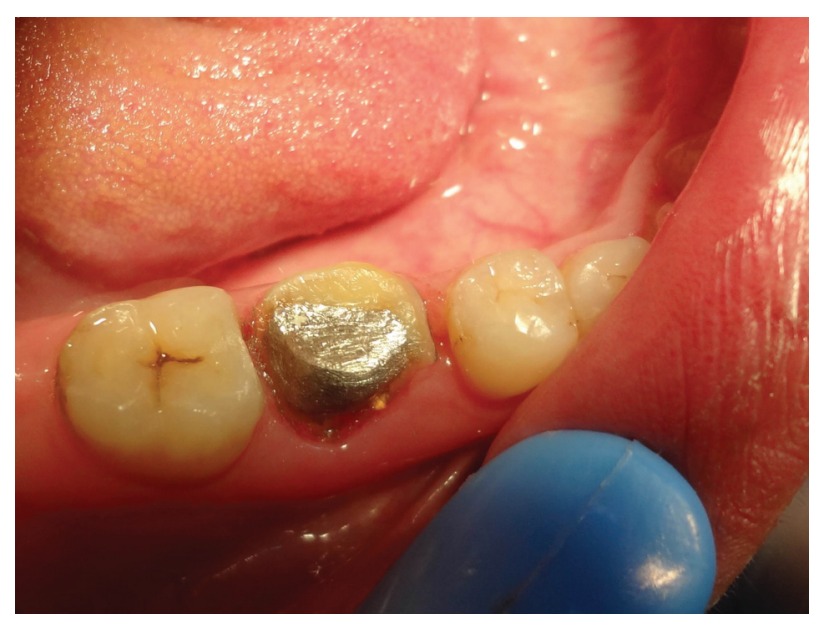

Figure 6: Metal post cementation 


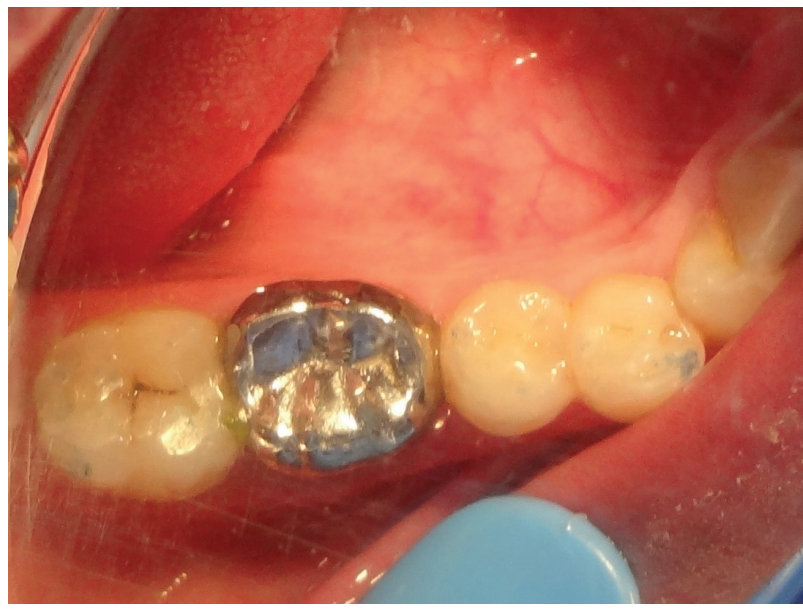

Figure 7: Metal Crown Cementation

\section{Discussion}

Post is usually required in anterior teeth requiring crown, whereas in molars pulp chamber and canals are usually adequate for the core retention. Molar with extensive coronal tooth destruction requires post and core. Rarely, more than one post is required in molars. ${ }^{1}$

Custom fitted post and core requires only a minimal reduction of root structure. If a cast post and core is used in cases of subgingival destruction, finish line for the crown can be prepared before post-and-core fabrication. This approach provides relatively easy access to subgingival areas. ${ }^{5}$ There are certain disadvantages of the cast post and core like high cost, lower retention, difficult temporization between appointments, risk of casting inaccuracies, additional removal of tooth structure, and greater number of visits. ${ }^{6}$ The clinician must judge every situation on its individual merits.

\section{Conclusion}

This article describes a direct technique for fabricating post and core patterns for restoration of mandibular first molar after endodontic treatment that had a heavily destructed clinical crown but with favorable root forms.

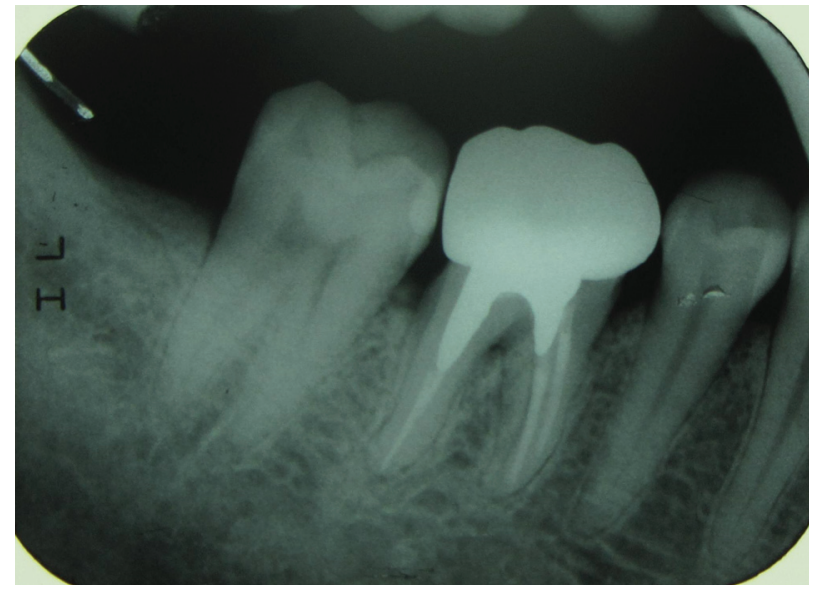

Figure 8: IOPA after metal post and crown cementation

\section{References}

1. Schwartz RS, Robbins JW. Post placement and restoration of endodontically treated teeth: a literature review. J Endod 2004;30(5):289-301.

2. Salvi GE, Siegrist Guldener BE, Amstad T, Joss A, Lang NP. Clinical evaluation of root filled teeth restored with or without post-andcore systems in a specialist practice setting. Int Endod J 2007;40(3):209-15.

3. Robbins JW. Guidelines for the restoration of endodontically treated teeth. J Am Dent Assoc 1990;120(5):558, 60, 62 passim.

4. Goodacre CJ, Spolnik KJ. The prosthodontic management of endodontically treated teeth: a literature review. Part I. Success and failure data, treatment concepts. J Prosthodont 1994;3(4):243-50.

5. Sadan A, Elliot R, Raigrodski AJ. Treatment planning extensively broken-down mandibular molars for post-and-core fabrication. Quintessence Int 1998;29(6):351-5.

6. Gogna R, Jagadish S, Shashikala K, Keshava Prasad B. Restoration of badly broken, endodontically treated posterior teeth. J Conserv Dent 2009;12(3):123-8.

7. Shillingburg HT, Jr., Fisher DW, Dewhirst RB. Restoration of endodontically treated posterior teeth. J Prosthet Dent 1970;24(4):401-9.

8. Bass EV. Cast post and core foundation for the badly broken down molar tooth. Aust Dent J 2002;47(1):57-62. 